\title{
Corrigendum: The oldest North American pachycephalosaurid and the hidden diversity of small-bodied ornithischian dinosaurs
}

David C. Evans, Ryan K. Schott, Derek W. Larson, Caleb M. Brown \& Michael J. Ryan

Nature Communications 4:1828 doi: 10.1038/ncomms2749 (2013); Published 7 May 2013; Updated 11 Feb 2014

In this Article, the museum catalogue numbers for the paratype and referred specimens of Acrotholus audeti nov. gen. et. sp. were inadvertently exchanged. The paratype reference should have been ROM 2964 and the catalogue number for the referred specimen should have been ROM 2962. 